\title{
Calculation of solute-vacancy binding energy in dilute fcc and bcc alloys by diffusion
}

\author{
D D PRUTHI \\ Chemistry Division, Bhabha Atomic Research Centre, Bombay 400085, India
}

\begin{abstract}
Abstrect. Due to excess charge of the solute with respect to solvent, the free eriergy of vacancy formation and migration in the neighbourhood of the solute will change. This results in a change in the solvent diffusivity. A relation for the solute vacancy binding energy for fcc and bcc lattices using enhancement factor has been derived considering the solute vacancy interactions to be limited to first neighbour and neglecting the changes in the solvent correlation factor.
\end{abstract}

Keywords. Solute-vacancy binding energy; diffusion; fec and bec alloys.

\section{Introduction}

In dilute solution solvent diffusivity $D(C)$ is linearly related to solute concentration (Pruthi and Agarwala 1982)

$$
D(C)=D(0)(1+b C),
$$

where $D(0)$ is the solvent diffusivity in pure solvent and $b$ is the enhancement factor.

From the atomistic point of view one could attribute changes in the solvent diffusivity to the excess field of solute atoms as compared to solvent atoms. This will change the vacancy concentration and will also perturb the vacancy solvent exchange frequency in its vicinity. In other words the free energies of formation and migration of vacancy in the neighbourhood of solute will be altered.

In figure 1, curve 1 shows the excess field due to the solute which is superimposed on the potential energy curve in the absence of solute viz curve 2, which results in the altered potential energy curve 3. $\Delta g_{1}$ and $\Delta g_{2}$ are the increase in the free energy of vacancy formation at sites 1 and 2 respectively and $\Delta g_{m}$ is the increase in the free energy at the saddle point. Therefore it should be possible to find a relation between the enhancement factor and free energy at the saddle point and near neighbour sites. One can hence calculate the free energy change at the 1 st nearest neighbour site (negative of which is solute vacancy binding energy) from the experimental values of the enhancement factor under sertain simplifying assumptions.

\section{Calculation of enhancement factor}

Solvent diffusivity in pure solvent and dilute alloy may be written as

$$
D(0)=\frac{1}{2} x^{2} \Gamma_{0} f_{0}
$$

and

$$
D(C)=\frac{1}{2} x^{2}\left(\sum_{i} \Gamma_{i} f_{i}+\Gamma_{f} f_{0}\right)
$$




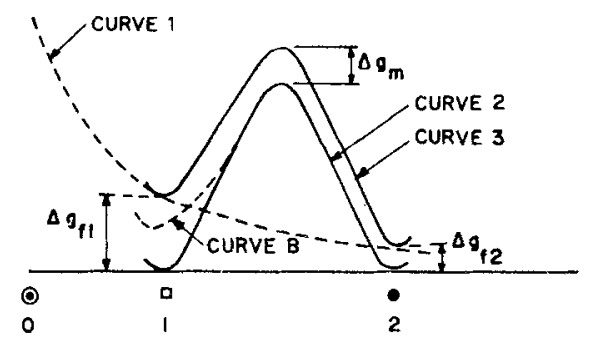

Figure 1. Potential energy curve for vacancy $(\square)$ solvent $(\bullet)$ exchange in the presence of solute $(\Theta)$.

where $x, \Gamma_{i}, \Gamma_{f}, f_{i}$ denote $x$ component of jump distance, number of $i$ type of jumps made per sec by the diffusing atom, free or unperturbed solvent jumps far away from the solute atom and correlation factor for $i$ type of jumps respectively. Subscript 0 stands for pure solvent. $\Gamma_{i}$ may be written as

$$
\Gamma_{i}=\sum_{j} C_{v i} W_{i j}
$$

where $C_{v i}$ is the probability of vacancy existing at sites of $i$ type (generally at $i$ th neighbour site of solute) and may be written as

$$
\begin{aligned}
C_{v i} & =C Z_{i} \exp -\left(g_{f}+\Delta g_{f i}\right) / k T, \\
& =C Z_{i} C_{v i}^{\prime},
\end{aligned}
$$

where $C$ is solute concentration, $Z_{i}$ is the number of $i$ th neighbours of solute, $C_{v i}$ is the probability of vacancy existing at the $i$ th neighbour sites of solute, $\Delta g_{f i}$ is the increase in the free energy of formation of solvent due to the presence of solute. $W_{i j}$ in (4) stands for the probability of vacancy at any of the ith neighbour site making $j$ type of jumps. It may be written as

$$
\begin{aligned}
W_{i j} & =Z_{i j} v \exp -\left(g_{m}+\Delta g_{m j}\right) / k T, \\
& =Z_{i j} W_{i j}^{\prime} .
\end{aligned}
$$

Here $Z_{i j}$ is the number of $j$ types of jumps which a vacancy at any of the $i$ th site can make with probability $W_{i j}, \Delta g_{m j}$ is the increase in the free energy of migration and $v$ is the vibrational frequency. Therefore $\Gamma_{i}$ may be written as

$$
\Gamma_{i}=\sum_{j} C Z_{i} Z_{i j} C_{v i}^{\prime} W_{i j}^{\prime}
$$

For pure solvent, vacancy can exist at any of the $Z_{0}$ neighbouring sites of a solvent atom (or a tracer atom). Therefore effective vacancy concentration and solvent jump frequency for pure solvent diffusion are

$$
\begin{aligned}
C_{v 0} & =Z_{0} \exp \left(-g_{f} / k T\right), \\
& =Z_{0} C_{v 0}^{\prime},
\end{aligned}
$$

and

$$
\Gamma_{0}=Z_{0} \Gamma_{0}^{\prime}
$$

In an alloy according to (7) and (3) $\sum_{i} \sum_{j} C Z_{i} Z_{i j}$ sites are involved in perturbed vacancy jumps. Therefore concentration of free vacancies and free vacancy jumps will 
be given by

$$
\begin{gathered}
C_{v f}=\left(Z_{0}-C \sum_{i} \sum_{j} Z_{i} Z_{i j}\right) C_{v 0}^{\prime} \\
\Gamma_{f}=\left(Z_{0}-C \sum_{i} \sum_{j} Z_{i} Z_{i j}\right) \Gamma_{0}^{\prime}
\end{gathered}
$$

For weak perturbation approximation, changes in solvent correlation factor for the perturbed jump frequencies are neglected, i.e. all $f_{i}$ are equal to $f_{0}$. Under this approximation (3) with the help of (2), (7) and (11) may be written as

$$
D(C)=D(0)\left[1+C \sum_{i} \sum_{j}\left(Z_{i} Z_{i j} / Z_{0}\right)\left(\Gamma_{i j}^{\prime} / \Gamma_{0}^{\prime}-1\right)\right]
$$

Therefore enhancement factor from (1) becomes

$$
\begin{aligned}
b & =(1 / C)[D(C) / D(0)-1], \\
& =\sum_{i} \sum_{j}\left(Z_{i} Z_{i j} / Z_{0}\right) \cdot\left(\Gamma_{i j}^{\prime} / \Gamma_{0}^{\prime}-1\right) .
\end{aligned}
$$

\subsection{Relation between dissociative and associative jumps}

It is shown below that the contributions from dissociative jumps and associative jumps are equal. In figure 1, if the vacancy is jumping from site 1 to site 2 ,

and

$$
C_{v 1}=\exp \left[-\left(g_{f}+\Delta g_{f 1}\right) / k T\right]
$$

$$
\begin{aligned}
& W_{12}=\exp \left[-\left(g_{m}-\Delta g_{f 1}+\Delta g_{m}\right) / k T\right], \\
& \therefore \Gamma_{12}^{\prime}=\Gamma_{0}^{\prime} \exp \left(-\Delta g_{m} / k T\right) .
\end{aligned}
$$

For $C_{w 2}$ and $W_{21}, \Delta g_{f 1}$ is replaced by $\Delta g_{f 2}$ and hence it gives the same result as (14).

If a vacancy can jump to $Z_{12}$ sites, for the reverse jumps it can be reached by the same number of jumps. Also if it can exist on $Z_{1}$ sites, near a solute atom, then the total number of sites involved for the forward and back ward jumps are $Z_{1} Z_{12}$. The latter i.e. the sites involved for the reverse jump may be written as $Z_{2} Z_{21}$. Hence $Z_{1} Z_{12}=Z_{2} Z_{21}$. Combining this with (14) shows that the contribution to $b$ for dissociative jumps and associative jumps are equal. In bcc structure there are eight first neighbours $\left(Z_{1}\right)$ and six second neighbours $\left(Z_{2}\right)$ of solute. Each first neighbour can jump to three second neighbour sites $\left(Z_{12}\right)$ and each second neighbour can jump to four first neighbour sites $\left(Z_{21}\right)$. Therefore dissociative jumps $(8 \times 3)$ are equal to associative jumps $(6 \times 4)$.

If the potential of the solute falls off in such a way that there is no change at the saddle point (e.g. curve $\mathbf{B}$ in figure 1), then $\Delta g_{m}$ is zero and therefore (14) gives $\Gamma_{i j}^{\prime}=\Gamma_{0}^{\prime}$ and hence contribution to $b$ (equation (13)) will be zero.

From (13), the expressions for $b$ derived by Lidiard (1960) for fcc lattice and LeClaire (1970) for bcc lattice can be written.

\section{Enhancement factor and change in free energy at the saddle point}

In the present discussion it will be assumed that solute vacancy interaction is limited up to first neighbours only. Hence there is no summation over $i$ in (13) and also $Z_{i}=Z_{0}$. 


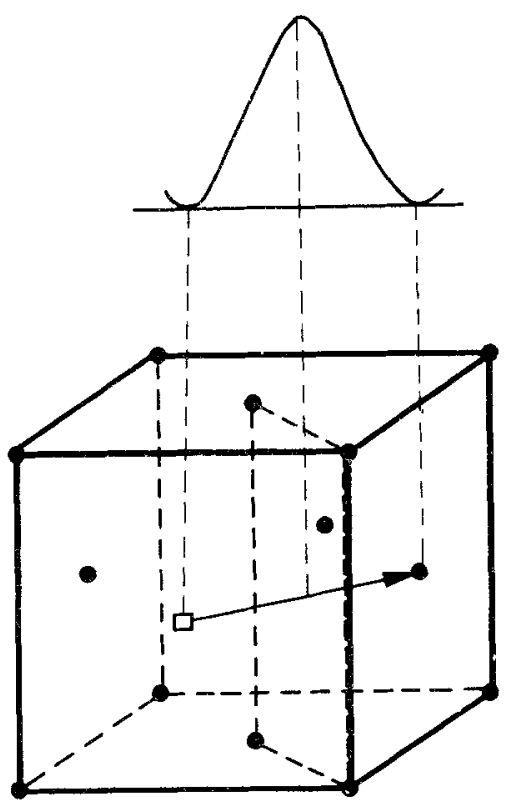

Figure 2. Potential energy curve for self diffusion in fcc lattice. Lattice atom and vacancy are shown as $(\bullet)$ and (口)

Therefore the expression for $b$ becomes

$$
b=\sum_{j} Z_{i j}\left(\Gamma_{i j}^{\prime} / \Gamma_{0}^{\prime}-1\right)
$$

For fcc structure according to figure 2, vacancy at first neighbour site of solute has four first, two second, four third and one fourth neighbour at distances of $\sqrt{0.5} a_{0}, a_{0}, \sqrt{1.5}$ $a_{0}$ and $\sqrt{2} a_{0}$ respectively. (Here $a_{0}$ is the lattice parameter). Their saddle points are at $\sqrt{6} a_{0} / 4, \sqrt{10} a_{0} / 4, \sqrt{14} a_{0} / 4$ and $\sqrt{18} a_{0} / 4$ respectively. The saddle point of the fourth neighbour lies at a distance greater than the second neighbours and hence will not contribute towards $b$. Using (15), the expression for $b$ may be written as

$$
\begin{aligned}
b= & 4 \exp \left(-\Delta g_{m 1} / k T\right)+4 \exp \left(-\Delta g_{m 2} / k T\right) \\
& +8 \exp \left(-\Delta g_{m 3} / k T\right)-16 .
\end{aligned}
$$

Here $\Delta g_{m 1}, \Delta g_{m 2}$ and $\Delta g_{m 3}$ are changes in the free energy at the saddle points for vacancy jumping to respectively, first, second and third neighbours. The factor 4 in the first term is due to the four first neighbours of vacancy, whereas for the second and third terms the values of 4 and 8 are double the number of second and third neighbours as both associative and dissociative jumps will contribute equally.

For bcc structure (figure 3), vacancy which is first neighbour of solute will have, three second, three third and one fifth neighbours. Their distances from the solute are $a_{0}$, $\sqrt{2} a_{0}$ and $\sqrt{3} a_{0}$ ( $a_{0}$ is the lattice parameter). In bcc structure the potential energy curve has got two humps as shown in figure 3 . The distances of first and second hump from the solute are respectively $\sqrt{24} a_{0} / 6, \sqrt{27} a_{0} / 6$ for second neighbour, $a_{0}, \sqrt{51}$ $a_{0} / 6$ for third neighbour and $\sqrt{48} a_{0} / 6, \sqrt{75} a_{0} / 6$ for fifth neighbour respectively. Saddle points of all the neighbours except second neighbour lie at a distance equal to (only for the first hump of third neighbour) or greater than the second neighbour. 


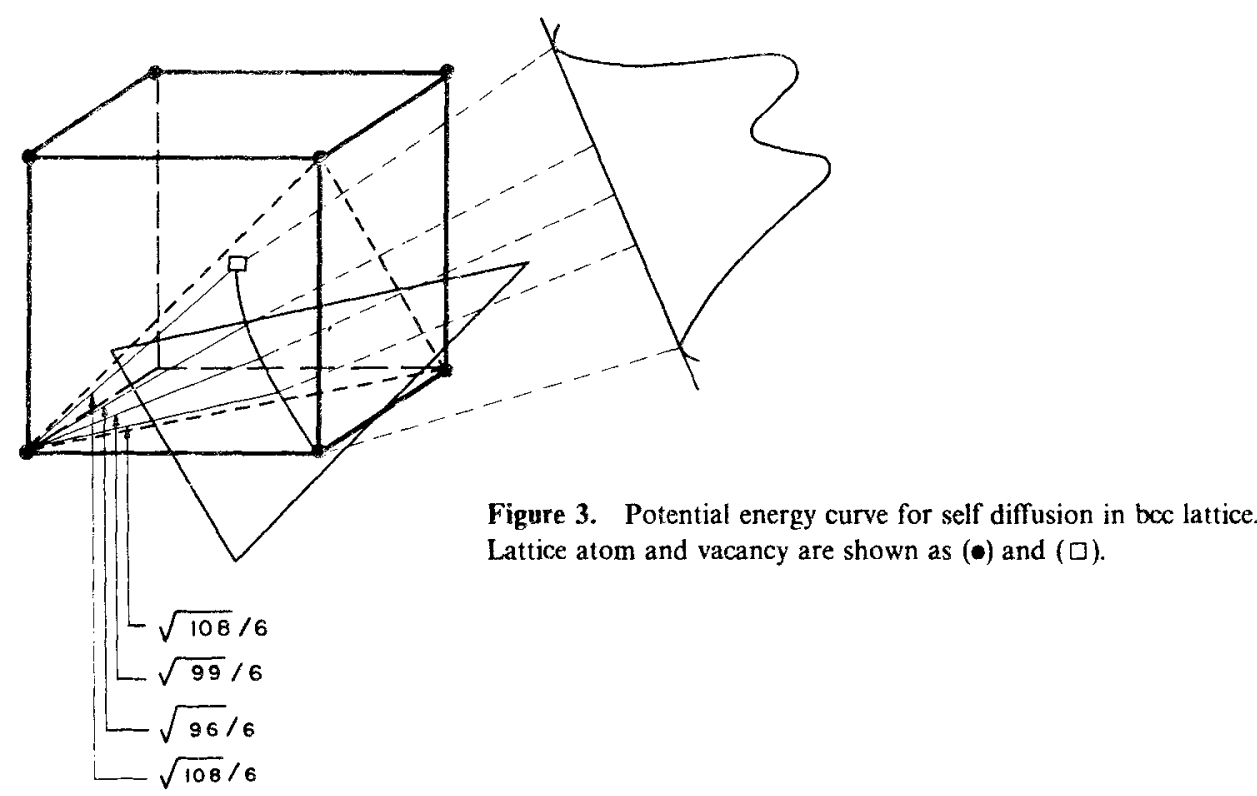

Therefore one need consider associative and dissociative jumps for second neighbour only. Equation (15) gives

$$
b=6\left[\exp \left(-\Delta g_{m} / k T\right)-1\right]
$$

\section{Calsulation of solute vacancy binding energy}

If it is assumed that excess potential due to solute dies down linearly from first neighbour site to second neighbour site and can be linearly extrapolated to the saddle point, then one can calculate solute vacancy binding energy.

For fcc structure $\Delta g_{m}$ may be written in terms of $\Delta g_{f}$ as

$$
\Delta g_{m i}=\frac{4-\sqrt{x_{i}}}{2(2-\sqrt{2})} \Delta g_{f},
$$

where $x_{1}, x_{2}$ and $x_{3}$ are 6,10 and 14 respectively. Hence $\Delta g_{f}$ may be calculated from the equation

$$
\begin{aligned}
b= & 4 \exp \left(-\alpha_{1} \Delta g_{f} / R T\right)+4 \exp \left(-\alpha_{2} \Delta g_{f} / R T\right) \\
& +8 \exp \left(-\alpha_{3} \Delta g_{f} / R T\right)-16 .
\end{aligned}
$$

Here free energy has been written per mole in place of per atom and $\alpha_{1}, \alpha_{2}, \alpha_{3}$ are coefficients of $\Delta g_{f}$ in (18) (viz. $\alpha_{1}=1.3234, \alpha_{2}=0.7150, \alpha_{3}=0.221$ ). The above equation shows that $\Delta g_{f}$ will depend on temperature. However (18) shows that the values of $\Delta g_{m 2}$ and $\Delta g_{m 3}$ are small as compared to $\Delta g_{m 1}$, hence the contribution from the two terms containing $\alpha_{2}$ and $\alpha_{3}$ will be small. If this contribution is neglected i.e. $\alpha_{2}=\alpha_{3}=0$ in (19) one gets the simple relation

$$
-\Delta g_{f}=\frac{R T}{\alpha_{1}} \log (1+b / 4)
$$


If the contribution from these two terms is not neglected one can use the following method of iteration to calculate the value of $\Delta g_{f}$. Let

then

$$
A=b+16-4 \exp \left(-\alpha_{2} \Delta g_{f} / R T\right)-8 \exp \left(-\alpha_{3} \Delta g_{f} / R T\right)
$$

$$
\Delta g_{f}=\frac{R T}{\alpha_{1}} \log (A / 4)
$$

First assume $\Delta g_{f}=0$ in (21) and substitute the value of $A$ in (22) to get $\Delta g_{f}$ which is then substituted in equation (21) to get a new value of $A$ which on substitution in (22) gives a new value of $\Delta g_{f}$. This process is repeated till the old and new value of $\Delta g_{f}$ agree.

For bcc lattice the potential energy curve has two humps. For solute vacancy attraction the second hump which is at the same distance as the first neighbour will be higher and will determine the potential barrier for the jump. Therefore $\Delta g_{f}=\Delta g_{m}$. Using (17) the free energy per mole can be written as

$$
-\Delta g_{f}=R T \log (1+b / 6)
$$

For repulsive interaction between solute and vacancy the potential of the first hump will be higher and hence it will determine the potential barrier for vacancy jump. Extrapolating linearly $\Delta g_{m}$ to $\Delta g_{f}$ one gets

$$
\begin{aligned}
\Delta g_{f} & =\frac{2 \sqrt{3}-3}{2 \sqrt{3}-2 \sqrt{2}} \Delta g_{m}, \\
& =0.73 R T \log (1+b / 6) .
\end{aligned}
$$

\section{Application}

Application to a few fcc systems at $730^{\circ} \mathrm{C}$ for different solutes in silver is shown in table 1 . It can be seen from the table that the values of solute vacancy binding energy increase as the value of enhancement factor increases. The contributions from second and third neighbours cannot be neglected when the value of $b$ is low. In table 2 , the values of solute vacancy binding energy for some bcc systems at one typical temperature are given.

Both tables show that the value of solute vacancy binding energy is of the order of a few kilo calories per mole in agreement with other studies (Anand and Agarwala 1972; Doyama 1978).

Table 1. Solute vacancy binding energy in some silver alloys at $1003 \mathrm{~K}$.

\begin{tabular}{lccc}
\hline & & \multicolumn{1}{c}{$\begin{array}{c}\Delta g_{f} \text { (in kcal/mol), contributions } \\
\text { from second and third neighbour }\end{array}$} \\
\cline { 3 - 4 } & & neglected & not neglected \\
\hline Solute & $b$ & -0.54 & -0.256 \\
$\mathrm{Au}$ & -1.2 & 1.52 & 1.07 \\
$\mathrm{Cd}$ & 7 & 2.18 & 1.67 \\
$\mathrm{In}$ & 13 & 3.51 & 3.03 \\
$\mathrm{Tl}$ & 37 & 3.97 & 3.54 \\
$\mathrm{Sb}$ & 52 & 4.70 & 4.33 \\
$\mathrm{~Pb}$ & 87 & & \\
\hline
\end{tabular}


Table 2. Solute vacancy binding energies in some bcc systems at one typical temperature.

\begin{tabular}{|c|c|c|c|}
\hline $\begin{array}{c}\text { System } \\
\text { solvent-solute }\end{array}$ & $\begin{array}{l}\text { Temp. } \\
(\mathbf{K})\end{array}$ & $b$ & $\begin{array}{c}-\Delta g_{f} \\
\text { (in kcal } / \mathrm{mol} \text { ) }\end{array}$ \\
\hline $\mathrm{Fe}-\mathrm{Co}$ & 1168 & $-1 \cdot 1$ & -0.34 \\
\hline$T i-V$ & 1373 & $-3 \cdot 7$ & -1.91 \\
\hline V-Ti & 1373 & $28 \cdot 7$ & $4 \cdot 79$ \\
\hline $\mathrm{Zr}-\mathrm{V}$ & 1167 & $-4 \cdot 1$ & -1.95 \\
\hline $\mathrm{V}-\mathrm{Zr}$ & 1578 & $28 \cdot 7$ & 5.50 \\
\hline $\mathrm{Zr}-\mathrm{Mn}$ & 1173 & $19 \cdot 0$ & 3.33 \\
\hline $\mathrm{V}-\mathrm{Fe}$ & 1573 & $18 \cdot 7$ & 4.42 \\
\hline V-Ta & 1960 & 1.7 & 0.97 \\
\hline $\mathrm{Fe}-\mathrm{Si}$ & 1400 & $4 \cdot 4$ & 4.51 \\
\hline
\end{tabular}

\section{Conclusion}

It is found that the changes in the free energy of the activated complex determine the change in solvent diffusivity rather than solute vacancy binding energy. If solute vacancy interaction is limited to first neighbours only, then its value can be calculated from simple relations between the solute vacancy binding energy and enhancement factor.

\section{References}

Anand M S and Agarwala R P 1972 Philos. Mag. 26297

LeClaire A D 1970 Philos. Mag. 21819

Lidiard A B 1960 Philos. Mag. 51171

Doyama M $1978 \mathrm{~J}$. Nucl. Mater. 69 and 70350

Pruthi D D and Agarwala R P 1982 Philos. Mag. 46841 